Pure and Applied Mathematics Quarterly

Volume 7, Number 4

(Special Issue:

In memory of Eckart Viehweg)

$1427-1447,2011$

\title{
Semipositivity Theorem for Reducible Algebraic Fiber Spaces
}

\author{
Yujiro Kawamata \\ Dedicated to the Memory of Eckart Viehweg
}

\begin{abstract}
We shall prove an extension of the semipositivity theorem for the case of reducible algebraic fiber spaces.
\end{abstract}

Keywords: Hodge theory, algebraic fiber space.

\section{INTRODUCTION}

The canonical divisor is an important invariant of an algebraic variety and reflects its geometric properties as an algebro-geometric curvature. When we compare canonical divisors of related varieties in many different situations, then we always find certain inequalities. This phenomena is not symmetric and called the positivity of the canonical divisor.

This paper is concerned with the positivity of canonical divisors for algebraic fiber spaces whose fibers are not necessarily irreducible. The irreducible case was considered in [9] and [10], where a positivity theorem was shown as an ingredient in the proof of a subadditivity theorem of the Kodaira dimension and a subadjunction theorem for higher codimensional subvarieties, respectively. This kind of positivity theorem is one of the main geometric applications of the Hodge theory. There is a metric version of the positivity theorem by Berndtson and Paun [2].

Received: Sept. 29, 2010; Revised: Jan. 18, 2011. 
We consider in this paper an extended case where the total space of an algebraic fiber space is non-normal, and prove the existence of a variations of mixed Hodge structures adapted to our situation by extending an argument in the article [9] which considered irreducible algebraic fiber spaces.

We fix the terminology. A reduced equi-dimensional algebraic scheme $X$ is said to be a simple normal crossing variety if it has smooth irreducible components and only normal crossing singularities. A closed stratum of $X$ is an irreducible component of the intersection of some of the irreducible components of $X$. Thus an irreducible component of $X$ is a closed stratum, and a closed stratum is a smooth variety.

Let $B$ be a reduced Cartier divisor on a simple normal crossing variety $X$. The pair $(X, B)$ is said to be a simple normal crossing pair if the following conditions are satisfied:

- $B$ does not contain any closed stratum of $X$.

- Let $Z$ be a closed stratum of $X$. Then the union $B_{Z}$ of the intersection $B \cap Z$ and all the closed strata properly contained in $Z$ is a simple normal crossing divisor on $Z$.

A closed stratum of the pair $(X, B)$ is either a closed stratum $Z$ of $X$ or an irreducible component of the intersection of some of the irreducible components of the divisor $B_{Z}$.

A locally free sheaf $F$ on a complete variety $Y$ is said to be numerically semipositive if the tautological invertible sheaf $\mathcal{O}_{\mathbf{P}(F)}(1)$ on the projectivized bundle $\mathbf{P}(F)$ is nef. This is a weaker condition than the existence of semi-positive metric, which is still weaker than the condition that the sheaf is generated by global sections.

We work over $\mathbf{C}$. We state the main theorem of this paper:

Theorem 1.1. Let $X$ be a projective simple normal crossing variety, $Y$ a smooth projective irreducible variety, and $f: X \rightarrow Y$ a projective surjective morphism. Let $B$ be a reduced Cartier divisor on $X$ such that $(X, B)$ is a simple normal crossing pair, and $C$ a simple normal crossing divisor on $Y$. Let $q$ be a nonnegative integer. Assume the following conditions: 
- Let $Z$ be a closed stratum of the pair $(X, B)$. Then the induced morphism $\left.f\right|_{Z}: Z \rightarrow Y$ is surjective, has connected fibers, and is smooth over $Y \backslash C$.

- If $Z$ is any closed stratum, then the local monodromies of the local system

$$
R^{d(Z / Y)+q}\left(\left.f\right|_{Z^{o}}\right)_{*} \mathbf{Z}_{Z^{\circ}}
$$

around the branches of $C$ are unipotent, where $d(Z / Y)=\operatorname{dim} Z-\operatorname{dim} Y$ denotes the fiber dimension of $\left.f\right|_{Z}$, and $Z^{o}=Z \backslash\left(\left.f\right|_{Z}\right)^{-1}(C)$.

Then the sheaf $R^{q} f_{*} \omega_{X / Y}(B)$ is locally free over $Y$ and is numerically semipositive, where $\omega_{X / Y}(B)$ is the relative canonical sheaf with logarithmic poles along $B$.

This result was proved if $X$ is irreducible in [5] after preceding results by [6] in the case $q=0, B=0$ and $\operatorname{dim} Y=1$, by [9] in the case $q=0$ and $B=0$, and by [12] and [13] in the case $B=0$.

The main result can be extended without change of the proof to the case where $X$ is a Kähler space, because we use only the fact that the cohomology groups of compact Kähler manifolds underly Hodge structures. The polarizations are defined over $\mathbf{R}$ instead of $\mathbf{Q}$, so we have to consider variations of mixed Hodge $\mathbf{R}$-structures in this case.

We use the strategy of [9] for the proof. We construct a variation of mixed Hodge structures on the open part $Y \backslash C$, and prove that the sheaf $R^{q} f_{*} \omega_{X / Y}(B)$ coincides with the canonical extensions of the graded piece of the highest degree with respect to the Hodge filtration. Then the semipositivity theorem follows from a result in [9], which is a consequence of Griffiths' result [8] on the semipositivity of the metric of the Hodge bundle over the open part $Y \backslash C$ together with considerations at the boundary $C$ using results of Schmid [14].

The local monodromies are always quasi-unipotent. Therefore the assumption on the local monodromies in the theorem is easily achieved if we replace $Y$ by a Kummer covering and change $X$ accordingly. This is the unipotent reduction theorem in [9]. In other words, it can be said that the semipositivity theorem holds for orbibundles on the orbifold above $Y$.

If the fiber space is irreducible, then we have a weak semistable reduction theorem of Abramovich and Karu [1], and we can analyse the mixed Hodge structures on the degenerate fibers more explicitly as in [11]. But we do not 
know whether there is an extension of the weak semistable reduction theorem to the case of reducible algebraic fiber spaces.

Although our result is stated in a numerical language, the proof of the semipositivity theorem yields a result that is stronger than a mere numerical statement; namely the Hodge bundle carries a semipositive singular hermitian metric whose singularities have no contributions to the curvature form.

We note that the underlying local system of the variation of mixed Hodge structures does not come from a locally trivial family of topological spaces, e.g., fibers of $f$ over $Y \backslash C$, but something virtually corresponding to their duals.

The contents of this paper is as follows. We recall the terminology on the cohomological mixed Hodge complexes and variations of mixed Hodge structures in $\S 2$. We start with the construction of the mixed Hodge structures on the general fibers in $\S 3$, and then make it relative over the base in $\S 4$. The main result is proved in $\S 5$.

We thank Valery Alexeev and Christopher Hacon whose questions, posed at the MSRI in Berkeley, were the starting point of the project. We also thank the MSRI for excellent working conditions.

\section{Preliminaries}

2.1. Cohomological mixed Hodge complexes. We recall the definition of cohomological mixed Hodge complexes in [3].

(1) [3] 8.1.1. A Hodge Q-complex of weight $n$

$$
H=\left(H_{\mathbf{Q}},\left(H_{\mathbf{C}}, F\right), \alpha\right)
$$

consists of the following data:

- a complex of $\mathbf{Q}$-vector spaces $H_{\mathbf{Q}} \in \mathrm{Ob} D^{+}(\mathbf{Q})$ which is bounded from below and such that the cohomologies $H^{k}\left(H_{\mathbf{Q}}\right)$ are finite-dimensional Qvector spaces for all $k$;

- a filtered complex $\left(H_{\mathbf{C}}, F\right) \in \mathrm{Ob} D^{+} F(\mathbf{C})$ which is bounded from below with an isomorphism $\alpha: H_{\mathbf{C}} \cong H_{\mathbf{Q}} \otimes \mathbf{C}$ in $D^{+}(\mathbf{C})$; 
such that the spectral sequence associated to the filtration $F$

$$
E_{1}^{p, q}=H^{p+q}\left(\operatorname{Gr}_{F}^{p}\left(H_{\mathbf{C}}\right)\right) \Rightarrow H^{p+q}\left(H_{\mathbf{C}}\right)
$$

degenerate at $E_{1}$, and

$$
\left(H^{k}\left(H_{\mathbf{Q}}\right),\left(H^{k}\left(H_{\mathbf{C}}\right), F\right)\right)
$$

with an isomorphism $H^{k}(\alpha): H^{k}\left(H_{\mathbf{C}}\right) \cong H^{k}\left(H_{\mathbf{Q}}\right) \otimes \mathbf{C}$ is a Hodge Q-structure of weight $n+k$.

Proposition 2.1. [3] 8.1.4. If $H$ is a Hodge Q-complex of weight $n$, then $H[m]$ is a Hodge $\mathbf{Q}$-complex of weight $m+n$.

(2) [3] 8.1.2. A cohomological Hodge Q-complex of weight $n$ on a topological space $X$

$$
H=\left(H_{\mathbf{Q}},\left(H_{\mathbf{C}}, F\right), \alpha\right)
$$

consists of the following data:

- a complex $H_{\mathbf{Q}} \in \mathrm{Ob} D^{+}(X, \mathbf{Q})$ of locally constant sheaves on $X$ which is bounded from below;

- a filtered complex $\left(H_{\mathbf{C}}, F\right) \in \mathrm{Ob} D^{+} F(X, \mathbf{C})$ of sheaves on $X$ which is bounded from below with an isomorphism $\alpha: H_{\mathbf{C}} \cong H_{\mathbf{Q}} \otimes \mathbf{C}$ in $D^{+}(X, \mathbf{C})$;

such that the triple

$$
\left(R \Gamma\left(H_{\mathbf{Q}}\right), R \Gamma\left(\left(H_{\mathbf{C}}, F\right)\right), R \Gamma(\alpha)\right)
$$

is a Hodge $\mathbf{Q}$-complex of weight $n$.

(3) [3] 8.1.5. A mixed Hodge Q-complex

$$
H=\left(\left(H_{\mathbf{Q}}, W\right),\left(H_{\mathbf{C}}, W, F\right), \alpha\right)
$$

consists of the following data:

- a filtered complex of $\mathbf{Q}$-vector spaces $\left(H_{\mathbf{Q}}, W\right) \in \mathrm{Ob} D^{+} F(\mathbf{Q})$ which is bounded from below with an increasing filtration $W$ such that the cohomologies $H^{k}\left(H_{\mathbf{Q}}\right)$ are finite-dimensional $\mathbf{Q}$-vector spaces for all $k$;

- a bifiltered complex $\left(H_{\mathbf{C}}, W, F\right) \in \mathrm{Ob} D^{+} F_{2}(\mathbf{C})$ which is bounded from below with an isomorphism $\alpha:\left(H_{\mathbf{C}}, W\right) \cong\left(H_{\mathbf{Q}}, W\right) \otimes \mathbf{C}$ in $D^{+} F(\mathbf{C})$; 
such that

$$
\left(\mathrm{Gr}_{n}^{W}\left(H_{\mathbf{Q}}\right),\left(\mathrm{Gr}_{n}^{W}\left(H_{\mathbf{C}}\right), F\right)\right)
$$

with an isomorphism

$$
\operatorname{Gr}_{n}^{W}(\alpha): \operatorname{Gr}_{n}^{W}\left(H_{\mathbf{C}}\right) \cong \operatorname{Gr}_{n}^{W}\left(H_{\mathbf{Q}}\right) \otimes \mathbf{C}
$$

is a Hodge $\mathbf{Q}$-complex of weight $n$ for any $n$.

(4) [3] 8.1.6. A cohomological mixed Hodge Q-complex on a topological space $X$

$$
H=\left(\left(H_{\mathbf{Q}}, W\right),\left(H_{\mathbf{C}}, W, F\right), \alpha\right)
$$

consists of the following data:

- a filtered complex $\left(H_{\mathbf{Q}}, W\right) \in \mathrm{Ob} D^{+} F(X, \mathbf{Q})$ which is bounded from below with an increasing filtration $W$ such that the $H^{k}\left(X, H_{\mathbf{Q}}\right)$ are finitedimensional Q-vector spaces;

- a bifiltered complex $\left(H_{\mathbf{C}}, W, F\right) \in \mathrm{Ob} D^{+} F_{2}(X, \mathbf{C})$ which is bounded from below with an isomorphism $\alpha:\left(H_{\mathbf{C}}, W\right) \cong\left(H_{\mathbf{Q}}, W\right) \otimes \mathbf{C}$ in $D^{+} F(X, \mathbf{C})$;

such that

$$
\left(\operatorname{Gr}_{n}^{W}\left(H_{\mathbf{Q}}\right),\left(\operatorname{Gr}_{n}^{W}\left(H_{\mathbf{C}}\right), F\right)\right)
$$

with an isomorphism

$$
\operatorname{Gr}_{n}^{W}(\alpha): \operatorname{Gr}_{n}^{W}\left(H_{\mathbf{C}}\right) \cong \operatorname{Gr}_{n}^{W}\left(H_{\mathbf{Q}}\right) \otimes \mathbf{C}
$$

is a cohomological Hodge Q-complex of weight $n$ for any $n$.

Proposition 2.2. [3] 8.1.8. If $X$ is a proper smooth variety and $B$ is a normal crossing divisor, then

$$
\left(\left(R i_{*} \mathbf{Q}_{X \backslash B}, \tau\right),\left(\Omega_{X}^{\bullet}(\log B), W, \sigma\right)\right)
$$

is a cohomological mixed Hodge $\mathbf{Q}$-complex on $X$, where $i: X \backslash B \rightarrow X$ is an open immersion, $\tau$ is a canonical filtration, $W$ is the filtration with respect to the order of $\log$ poles, and $\sigma$ is a stupid filtration.

Proposition 2.3. [3] 8.1.7. If $H=\left(\left(H_{\mathbf{Q}}, W\right),\left(H_{\mathbf{C}}, W, F\right)\right)$ is a cohomological mixed Hodge $\mathbf{Q}$-complex, then $R \Gamma H=\left(R \Gamma\left(H_{\mathbf{Q}}, W\right), R \Gamma\left(H_{\mathbf{C}}, W, F\right)\right)$ is a mixed Hodge Q-complex. 
Theorem 2.4. [3] 8.1.9. Let $H=\left(\left(H_{\mathbf{Q}}, W\right),\left(H_{\mathbf{C}}, W, F\right), \alpha\right)$ be a mixed Hodge Q-complex. Then the following hold:

(1) $H^{n}\left(H_{\mathbf{Q}}\right)=\left(\left(H^{n}\left(H_{\mathbf{Q}}\right), W[n]\right),\left(H^{n}\left(H_{\mathbf{C}}\right), W[n], F\right), H^{n}(\alpha)\right)$ is a mixed Hodge $\mathbf{Q}$-structure.

(2) The spectral sequence defined by the weight filtration

$$
{ }_{W} E_{1}^{p, q}=H^{p+q}\left(G r_{-p}^{W}\left(H_{\mathbf{Q}}\right)\right) \Rightarrow H^{p+q}\left(H_{\mathbf{Q}}\right)
$$

degenerates at $E_{2}$.

(3) The spectral sequence defined by the Hodge filtration

$$
{ }_{F} E_{1}^{p, q}=H^{p+q}\left(G r_{F}^{p}\left(H_{\mathbf{C}}\right)\right) \Rightarrow H^{p+q}\left(H_{\mathbf{C}}\right)
$$

degenerates at $E_{1}$.

2.2. Variations of mixed Hodge structures. A variation of mixed Hodge Q-structures on a complex manifold $Y$

$$
H=\left(\left(H_{\mathbf{Q}}, W\right),(\mathcal{H}, W, F), \alpha\right)
$$

consists of the following data:

- a filtered locally constant sheaf of $\mathbf{Q}$-modules of finite rank $\left(H_{\mathbf{Q}}, W\right) \in$ Ob $D^{+}(Y, \mathbf{Q})$ with an increasing filtration $W$ by locally constant sheaves;

- a bifiltered locally free sheaf of coherent $\mathcal{O}_{Y \text {-modules }}(\mathcal{H}, W, F) \in$ Ob $D^{+} F_{2}\left(X, \mathcal{O}_{Y}\right)$ by an increasing filtration $W$ by locally free sheaves with an isomorphism $\alpha:(\mathcal{H}, W) \cong\left(H_{\mathbf{Q}}, W\right) \otimes \mathcal{O}_{Y}$ in $D^{+} F\left(X, \mathcal{O}_{Y}\right)$, and a decreasing filtration $F$ by locally free sheaves

such that the natural connection $\nabla$ on $\mathcal{H}$ induced from $\alpha$ satisfies

$$
\nabla: F^{p} \mathcal{H} \rightarrow F^{p-1} \mathcal{H} \otimes \Omega_{Y}^{1}
$$

and, for each point $y \in Y, H_{y}=\left(\left(H_{\mathbf{Q}, y}, W\right),\left(\mathcal{H} \otimes \mathcal{O}_{y}, W, F\right), \alpha\right)$ is a mixed Hodge Q-structure.

A variation of mixed Hodge $\mathbf{Q}$-structures $H$ is said to be graded polarizable if $\operatorname{Gr}_{q}^{W}(H)$ are polarized variations of Hodge Q-structures for all $q$ [15].

Let $H=\left(H_{\mathbf{Q}},(\mathcal{H}, F)\right)$ be a polarized variation of (pure) Hodge $\mathbf{Q}$-structures. By [8] Theorem 5.2, if $d$ is the largest number such that $F^{d} \mathcal{H} \neq 0$, then the 
curvature form $\Theta$ of the Hodge bundle $F^{d} \mathcal{H}$ is given by the formula

$$
\left(\Theta e, e^{\prime}\right)=\left(\sigma(e), \sigma\left(e^{\prime}\right)\right)
$$

where $\sigma: F^{d} \mathcal{H} \rightarrow F^{d-1} \mathcal{H} / F^{d} \mathcal{H} \otimes \Omega_{Y}^{1}$ is induced from the connection $\nabla$ of the flat bundle $\mathcal{H}$ and the inner product is given by the polarization. In particular, the curvature $\Theta$ is semipositive differential form of type $(1,1)$.

Let $H$ be a variation of mixed Hodge $\mathbf{Q}$-structures over $Y$, and assume that $Y$ is embedded into a complex manifold $\tilde{Y}$ such that the complement $C=\tilde{Y} \backslash Y$ is a normal crossing divisor. Assuming that the local monodromies of the local system $H_{\mathbf{Q}}$ around the branches of $C$ are unipotent, we define a canonical extension of $H$ as follows. Let $y \in C$ be an arbitrary point at the boundary, let $N_{i}$ be the logarithm of the local monodromies around the branches of $C$ around $y$, and let $t_{i}$ be the local coordinates defining the branches. Then the canonical extension $\tilde{\mathcal{H}}$ is defined as a locally free sheaf on $\tilde{Y}$ which is generated by sections of the form $\tilde{v}=\exp \left(-\sum_{i} t_{i} N_{i} / 2 \pi \sqrt{-1}\right)(v)$ near $y$, where the $v$ are multi-valued flat sections of $H_{\mathbf{Q}}$. We note that the monodromy actions are canceled and the $\tilde{v}$ are single-valued holomorphic sections of $\mathcal{H}$ outside the boundary divisors.

The Hodge filtration $F$ of $\mathcal{H}$ extends to $\tilde{\mathcal{H}}$ such that $\operatorname{Gr}_{F}(\tilde{\mathcal{H}})$ is still a locally free sheaf on $\tilde{Y}$. Indeed this is a consequence of the nilpotent orbit theorem [14] when $H$ is a variation of pure Hodge structures, and the general case follows immediately from this.

\section{Absolute case}

In this subsection we do the calculation on the fibers of $f$ over $Y \backslash C$. In other words, we consider the case where $Y$ is a point. The purpose is also to explain the idea of the proof without too much notation.

Let $(X, B)$ be a projective simple normal crossing pair. We denote by $X^{[q]}$ the disjoint union of all the closed strata of $X$ which are of codimension $q$. For example, $X^{[0]}$ is the normalization of $X, X^{[1]}$ is the normalization of the double locus of $X$, and so on. We denote by $B^{[q]}$ the disjoint union of the $B_{Z}$ for all the irreducible components $Z$ of $X^{[q]}$. It is a simple normal crossing divisor on $X^{[q]}$. Let $i_{q}: X^{[q]} \backslash B^{[q]} \rightarrow X^{[q]}$ be the open immersions.

In the following, we identify sheaves with their direct images under finite morphisms by the abuse of notation. 
First we define the logarithmic De Rham complex $\tilde{\Omega}_{X}^{\bullet}(\log B)$ for the pair $(X, B)$ as the first term of the following exact sequence given by the residue homomorphisms

$$
\begin{aligned}
0 & \rightarrow \tilde{\Omega}_{X}^{\bullet}(\log B) \rightarrow \Omega_{X^{[0]}}^{\bullet}\left(\log B^{[0]}\right) \rightarrow \Omega_{X^{[1]}}^{\bullet}\left(\log B^{[1]}\right)[-1] \\
& \rightarrow \Omega_{X^{[2]}}^{\bullet}\left(\log B^{[2]}\right)[-2] \rightarrow \ldots
\end{aligned}
$$

For example

$$
\tilde{\Omega}_{X}^{\operatorname{dim} X}(\log B) \cong \omega_{X}(B)
$$

is an invertible sheaf, but

$$
\tilde{\Omega}_{X}^{0}(\log B) \cong \mathcal{O}_{X[0]}
$$

is the structure sheaf of the normalization of $X$.

If $X$ is embedded into a smooth variety $V$ as a simple normal crossing divisor, and if $B$ coincides with a restriction $B=W \cap X$ of another simple normal crossing divisor $W$ on $V$ such that $X \cup W$ is also a simple normal crossing divisor, then there is an exact sequence given by the residue homomorphism

$$
0 \rightarrow \Omega_{V}^{\bullet}(\log W) \rightarrow \Omega_{V}^{\bullet}(\log (X+W)) \rightarrow \tilde{\Omega}_{X}^{\bullet}(\log B)[-1] \rightarrow 0 .
$$

Our complex is different from the complex $\bar{\Omega}_{X}^{\bullet}(\log B)$ considered by Du Bois [4], which is a single complex associated to the following double complex coming from the Mayer-Vietoris exact sequence:

$$
\bar{\Omega}_{X}^{\bullet}(\log B)=\left\{\Omega_{X^{[0]}}^{\bullet}\left(\log B^{[0]}\right) \rightarrow \Omega_{X^{[1]}}^{\bullet}\left(\log B^{[1]}\right) \rightarrow \Omega_{X^{[2]}}^{\bullet}\left(\log B^{[2]}\right) \rightarrow \ldots\right\}
$$

where the arrows are the restriction homomorphisms. We note that both these complexes are different from the complex of Kähler differentials. We have $\bar{\Omega}_{X}^{0} \cong$ $\mathcal{O}_{X}$, and

$$
R i_{*} \mathbf{C}_{X \backslash B} \cong \bar{\Omega}_{X}^{\bullet}(\log B)
$$

for the open immersion $i: X \backslash B \rightarrow X$.

On the other hand, there is no obvious topological space which corresponds to the complex $\tilde{\Omega}_{X}^{\bullet}(\log B)$ in a similar way as in the case of Du Bois complex. In order to define an object on the Q-level, we consider closed strata $Z$ and $Z^{\prime}$ of $X$ such that $Z^{\prime}$ is contained in $Z$ as a prime divisor. Let $B_{Z}^{\prime}$ be the union of all the irreducible components of $B_{Z}$ except $Z^{\prime}$. Then we have $B_{Z^{\prime}}=B_{Z}^{\prime} \cap Z^{\prime}$. Let $i_{Z}: Z \backslash B_{Z} \rightarrow Z$ and $i_{Z^{\prime}}: Z^{\prime} \backslash B_{Z^{\prime}} \rightarrow Z^{\prime}$ be open immersions. We replace the 
residue homomorphism by the composition of the following morphisms between objects on $X$ :

$$
R\left(i_{Z}\right)_{*} \mathbf{Q}_{Z \backslash B_{Z}} \rightarrow R\left(i_{Z^{\prime}}\right)_{*} R \Gamma_{Z^{\prime} \backslash B_{Z^{\prime}}}\left(\mathbf{Q}_{Z \backslash B_{Z}^{\prime}}\right)[1] \cong R\left(i_{Z^{\prime}}\right)_{*} \mathbf{Q}_{Z^{\prime} \backslash B_{Z^{\prime}}}[-1]
$$

where the first morphism is induced from a distinguished triangle

$$
R \Gamma_{Z^{\prime} \backslash B_{Z^{\prime}}}\left(\mathbf{Q}_{Z \backslash B_{Z}^{\prime}}\right) \rightarrow \mathbf{Q}_{Z \backslash B_{Z}^{\prime}} \rightarrow \mathbf{Q}_{Z \backslash B_{Z}} \rightarrow R \Gamma_{Z^{\prime} \backslash B_{Z^{\prime}}}\left(\mathbf{Q}_{Z \backslash B_{Z}^{\prime}}\right)[1]
$$

and the second isomorphism follows from the fact that $Z \backslash B_{Z}^{\prime}$ is analytic locally isomorphic to an oriented disk bundle over $Z^{\prime} \backslash B_{Z^{\prime}}$ near $Z^{\prime} \backslash B_{Z^{\prime}}$. By taking the alternating sum, we obtain

$$
R\left(i_{q}\right)_{*} \mathbf{Q}_{X^{[q]} \backslash B^{[q]}} \rightarrow R\left(i_{q+1}\right)_{*} \mathbf{Q}_{X^{[q+1]} \backslash B^{[q+1]}}[-1]
$$

where the sign convention is defined as follows. Let $X_{i}(i=1, \ldots, s)$ be the irreducible components of $X$, and assume that $Z$ is an irreducible component of the intersection $\bigcap_{j=0}^{q} X_{i_{j}}$ for $i_{0}<\cdots<i_{q}$, while $Z^{\prime}$ is an irreducible component of the intersection $\bigcap_{j=0}^{q+1} X_{i_{j}}$. Then the sign is defined to be $\operatorname{sgn}\left(i_{0}, \ldots, i_{q}, i_{q+1}\right)$.

We recall the definition of a convolution of a complex of objects in a triangulated category [7]. This construction is similar to the reconstruction of a module with a finite filtration from the graded pieces. There are two ways of constructions, the bottom up and the top down. Let

$$
a_{n} \rightarrow a_{n-1} \rightarrow \cdots \rightarrow a_{1} \rightarrow a_{0}
$$

be a complex of objects. If there exists a sequence of distinguished triangles

$$
\begin{aligned}
& b_{k+1}[k+1] \rightarrow a_{k+1} \rightarrow b_{k}[k] \rightarrow b_{k+1}[k+2] \\
& \text { (resp. } \left.b_{k+1} \rightarrow a_{k} \rightarrow b_{k} \rightarrow b_{k+1}[1]\right)
\end{aligned}
$$

for $0 \leq k<n$ with an isomorphism $b_{0} \rightarrow a_{0}$ (resp. $a_{n} \rightarrow b_{n}$ ), then $b_{n}[n]$ (resp. $b_{0}$ ) is said to be a convolution of the complex from the right (resp. left). A convolution may not exist and may not be unique if it exists. But the two different constructions yield the same set of convolutions by virtue of the octahedral axiom. For example, if there is a finite filtration of a module

$$
F_{0} \subset F_{1} \subset \cdots \subset F_{n-1} \subset F_{n}
$$

then $F_{n}$ is a convolution of a complex

$$
\mathrm{Gr}_{n}^{F}[-n] \rightarrow \mathrm{Gr}_{n-1}^{F}[-n+1] \rightarrow \cdots \rightarrow \mathrm{Gr}_{1}^{F}[-1] \rightarrow \mathrm{Gr}_{0}^{F}
$$

We have the following substitute of the Mayer-Vietoris exact sequence: 
Lemma 3.1. There exists an object $H_{\mathbf{Q}}$ on $X$ as a convolution of the following complex:

$$
R\left(i_{0}\right)_{*} \mathbf{Q}_{X^{[0]} \backslash B^{[0]}} \rightarrow R\left(i_{1}\right)_{*} \mathbf{Q}_{X^{[1]} \backslash B^{[1]}}[-1] \rightarrow R\left(i_{2}\right)_{*} \mathbf{Q}_{X^{[2]} \backslash B^{[2]}}[-2] \rightarrow \ldots
$$

where the direct images of objects by closed immersions to $X$ are denoted by the same symbols, such that

$$
H_{\mathbf{Q}} \otimes \mathbf{C} \cong \tilde{\Omega}_{X}^{\bullet}(\log B) .
$$

Proof. We can check that the given sequence is a complex thanks to the sign convention above. We have isomorphisms

$$
\alpha_{q}: R\left(i_{q}\right)_{*} \mathbf{Q}_{X^{[q]} \backslash B[q]} \otimes \mathbf{C} \cong \Omega_{X^{[q]}}^{\bullet}\left(\log B^{[q]}\right) .
$$

which are compatible with the boundary morphisms of both complexes of objects.

Let $a_{n-k}=R\left(i_{k}\right)_{*} \mathbf{Q}_{X^{[k]} \backslash B^{[k]}}[-k]$ for $n=\operatorname{dim} X$ and $0 \leq k \leq n$. We execute the convolution process from the right. Let

$$
a_{n} \rightarrow a_{n-1} \rightarrow \cdots \rightarrow a_{k+1} \rightarrow b_{k}[k]
$$

be a complex at an intermediate step for $0 \leq k<n$. Then a complex of the next step is constructed in the following way. If

$$
b_{k+1}[k+1] \rightarrow a_{k+1} \rightarrow b_{k}[k] \rightarrow b_{k+1}[k+2]
$$

is a distinguished triangle, then the boundary morphism $a_{k+2} \rightarrow a_{k+1}$ is liftable to a morphism $a_{k+2} \rightarrow b_{k+1}[k+1]$ because its composition with the boundary morphism $a_{k+1} \rightarrow b_{k}[k]$ vanishes. If the composition $a_{k+3} \rightarrow a_{k+2} \rightarrow b_{k+1}[k+1]$ is zero, then we obtain a complex of the next step with shorter length.

If we tensorize everything in the above procedure with $\mathbf{C}$, then the corresponding complex at the intermediate step is given by

$$
\begin{aligned}
& a_{n-i} \otimes \mathbf{C} \cong \Omega_{X^{[i]}}^{\bullet}\left(\log B^{[k]}\right)[-i] \\
& b_{n-k}[n-k] \otimes \mathbf{C} \cong \operatorname{Ker}\left\{\Omega_{X^{[k]}}^{\bullet}\left(\log B^{[k]}\right)[-k] \rightarrow \Omega_{X^{[k+1]}}^{\bullet}\left(\log B^{[k+1]}\right)[-k-1]\right\}
\end{aligned}
$$

for $0 \leq i<k$. Then $a_{n-k+2} \otimes \mathbf{C}$ and $b_{n-k+1}[n-k+1] \otimes \mathbf{C}$ are respectively isomorphic to

$$
\begin{aligned}
& \Omega_{X^{[k-2]}}^{\bullet}\left(\log B^{[k-2]}\right)[-k+2] \\
& \operatorname{Ker}\left\{\Omega_{X^{[k-1]}}^{\bullet}\left(\log B^{[k-1]}\right)[-k+1] \rightarrow \Omega_{X^{[k]}}^{\bullet}\left(\log B^{[k]}\right)[-k]\right\} .
\end{aligned}
$$


Therefore the lifted morphism $a_{n-k+2} \otimes \mathbf{C} \rightarrow b_{n-k+1}[n-k+1] \otimes \mathbf{C}$ is uniquely determined by the compatibility with the given boundary morphism

$$
\Omega_{X^{[k-2]}}^{\bullet}\left(\log B^{[k-2]}\right)[-k+2] \rightarrow \Omega_{X^{[k-1]}}^{\bullet}\left(\log B^{[k-1]}\right)[-k+1] .
$$

It follows that the lifted morphism $a_{n-k+2} \rightarrow b_{n-k+1}[n-k+1]$ is also unique and gives a shortened complex of the next step yielding the desired convolution.

For example, if $X$ is embedded into a smooth variety $V$ with a simple normal crossing divisor $X \cup W$ such that $B=X \cap W$, then we have

$$
H_{\mathbf{Q}} \cong R \Gamma_{X} R i_{1 *} \mathbf{Q}_{V \backslash W}[2]
$$

where $i_{1}: V \backslash W \rightarrow V$ is an open immersion. Indeed we have distinguished triangles

$$
\begin{aligned}
& R \Gamma_{X} R i_{1 *} \mathbf{Q}_{V \backslash W} \rightarrow R i_{1 *} \mathbf{Q}_{V \backslash W} \rightarrow R i_{2 *} \mathbf{Q}_{V \backslash(X \cup W)} \rightarrow R \Gamma_{X} R i_{1 *} \mathbf{Q}_{V \backslash W}[1] \\
& \tilde{\Omega}_{X}^{\bullet}(\log B) \rightarrow \Omega_{V}^{\bullet}(\log W)[2] \rightarrow \Omega_{V}^{\bullet}(\log (X+W))[2] \rightarrow \tilde{\Omega}_{X}^{\bullet}(\log B)[1]
\end{aligned}
$$

where $i_{2}: V \backslash(X \cup W) \rightarrow V$ is another open immersion.

We define a weight filtration, an increasing filtration denoted by $W$, on the De Rham complex $\tilde{\Omega}_{X}^{\bullet}(\log B)$ by the following exact sequence

$$
\begin{aligned}
& 0 \rightarrow W_{q}\left(\tilde{\Omega}_{X}^{\bullet}(\log B)\right) \rightarrow W_{q}\left(\Omega_{X^{[0]}}^{\bullet}\left(\log B^{[0]}\right)\right) \\
& \rightarrow W_{q-1}\left(\Omega_{X^{[1]}}^{\bullet}\left(\log B^{[1]}\right)[-1]\right) \rightarrow W_{q-2}\left(\Omega_{X^{[2]}}^{\bullet}\left(\log B^{[2]}\right)[-2]\right) \rightarrow \ldots
\end{aligned}
$$

where the $W$ 's from the second terms denote the filtration with respect to the order of log poles. We define a Hodge filtration, a decreasing filtration denoted by $F$, by

$$
F^{p}\left(\tilde{\Omega}_{X}^{\bullet}(\log B)\right)=\tilde{\Omega}_{X}^{\geq p}(\log B)
$$

\section{Lemma 3.2.}

$$
\begin{aligned}
& \operatorname{Gr}_{q}^{W}\left(\tilde{\Omega}_{X}^{\bullet}(\log B)\right) \cong \bigoplus_{\operatorname{dim} X-\operatorname{dim} Z=q} \Omega_{Z}^{\bullet}[-q] \\
& F^{r}\left(G r_{q}^{W}\left(\tilde{\Omega}_{X}^{\bullet}(\log B)\right)\right) \cong \bigoplus_{\operatorname{dim} X-\operatorname{dim} Z=q} \Omega_{\bar{Z}}^{\geq r-q}[-q]
\end{aligned}
$$

where the $Z$ run all the closed strata of the pair $(X, B)$ of codimension $q$. 
Proof. Let $X^{[p],[q]}$ be the disjoint union of all the closed strata of the pair $(X, B)$ which are contained in $X^{[p]}$ and have codimension $q$ in $X^{[p]}$. Then the residue homomorphism gives an isomorphism

$$
\operatorname{Gr}_{q-p}^{W}\left(\Omega_{X[p]}^{\bullet}\left(\log B^{[p]}\right)\right) \cong \Omega_{X[p],[q-p]}^{\bullet}[-q+p] .
$$

Thus we obtain exact sequences

$$
0 \rightarrow \operatorname{Gr}_{q}^{W}\left(\tilde{\Omega}_{X}^{\bullet}(\log B)\right)[q] \rightarrow \Omega_{X[0],[q]}^{\bullet} \rightarrow \Omega_{X[1],[q-1]}^{\bullet} \rightarrow \Omega_{X[2],[q-2]}^{\bullet} \rightarrow \ldots
$$

Let $Z$ be an irreducible component of $X^{[p],[q-p]}$ but not of $X^{[p+1],[q-p-1]}$ for some $p \leq q$. Then $Z$ appears $\left(\begin{array}{c}p+1 \\ p^{\prime}+1\end{array}\right)$ times in $X^{\left[p^{\prime}\right],\left[q-p^{\prime}\right]}$ for $0 \leq p^{\prime} \leq p$. Therefore the contributions of $Z$ cancel except once.

We define a weight filtration $W_{q}\left(H_{\mathbf{Q}}\right)$ on the $\mathbf{Q}$-level object $H_{\mathbf{Q}}$ as a convolution of the following complex

$$
\begin{aligned}
& \tau_{\leq q}\left(R\left(i_{0}\right)_{*} \mathbf{Q}_{X^{[0]} \backslash B^{[0]}}\right) \rightarrow \tau_{\leq q}\left(R\left(i_{1}\right)_{*} \mathbf{Q}_{X^{[1]} \backslash B^{[1]}}[-1]\right) \\
& \rightarrow \tau_{\leq q}\left(R\left(i_{2}\right)_{*} \mathbf{Q}_{X^{[2]} \backslash B^{[2]}}[-2]\right) \rightarrow \ldots
\end{aligned}
$$

where $\tau$ denotes the canonical filtration such that there is an isomorphism

$$
W_{q}\left(H_{\mathbf{Q}}\right) \otimes \mathbf{C} \cong W_{q}\left(\tilde{\Omega}_{X}^{\bullet}(\log B)\right) .
$$

The existence of such a convolution is guaranteed by the same reason as before. Here we note that

$$
\tau_{\leq q}\left(R\left(i_{p}\right)_{*} \mathbf{Q}_{X^{[p]} \backslash B^{[p]}}[-p]\right)=\tau_{\leq q-p}\left(R\left(i_{p}\right)_{*} \mathbf{Q}_{X^{[p]} \backslash B^{[p]}}\right)[-p] .
$$

The "filtration" of the object $H_{\mathbf{Q}}$ means the following: $H_{\mathbf{Q}}$ is a convolution of a complex

$$
\rightarrow \mathrm{Gr}_{q+1}^{W}\left(H_{\mathbf{Q}}\right)[-q-1] \rightarrow \mathrm{Gr}_{q}^{W}\left(H_{\mathbf{Q}}\right)[-q] \rightarrow \mathrm{Gr}_{q-1}^{W}\left(H_{\mathbf{Q}}\right)[-q+1] \rightarrow \ldots
$$

where the objects $W_{q}\left(H_{\mathbf{Q}}\right)$ and $\mathrm{Gr}_{q}^{W}\left(H_{\mathbf{Q}}\right)$ appear in the intermediate steps of the convolution process in the following distinguished triangles

$$
W_{q-1}\left(H_{\mathbf{Q}}\right) \rightarrow W_{q}\left(H_{\mathbf{Q}}\right) \rightarrow \operatorname{Gr}_{q}^{W}\left(H_{\mathbf{Q}}\right) \rightarrow W_{q-1}\left(H_{\mathbf{Q}}\right)[1] .
$$

By the same argument as before, we obtain

$$
\mathrm{Gr}_{q}^{W}\left(H_{\mathbf{Q}}\right) \cong \bigoplus_{\operatorname{dim} X-\operatorname{dim} Z=q} \mathbf{Q}_{Z}[-q]
$$

Hence we have the following: 


\section{Theorem 3.3.}

$$
\left(\left(H_{\mathbf{Q}}, W\right),\left(\tilde{\Omega}_{X}^{\bullet}(\log B), W, F\right)\right)
$$

is a cohomological mixed Hodge $\mathbf{Q}$-complex on $X$.

Proof. If we define a shifted filtration $F(-q)$ on $\Omega_{Z}^{\bullet}$ by $F(-q)^{r}=F^{r-q}$, then

$$
\left(\mathbf{Q}_{Z}, \Omega_{Z}^{\bullet}, F(-q)\right)
$$

is a cohomological Hodge $\mathbf{Q}$-complex of weight $2 q$. Hence

$$
\left(\mathbf{Q}_{Z}[-q], \Omega_{Z}^{\bullet}[-q], F(-q)[-q]\right)
$$

is a cohomological Hodge $\mathbf{Q}$-complex of weight $q$.

Corollary 3.4. The weight spectral sequence

$$
{ }_{W} E_{1}^{p, q}=H^{p+q}\left(X, G r_{-p}^{W}\left(H_{\mathbf{Q}}\right)\right) \Rightarrow H^{p+q}\left(H_{\mathbf{Q}}\right)
$$

degenerates at $E_{2}$, the Hodge spectral sequence

$$
{ }_{F} E_{1}^{p, q}=H^{q}\left(X,\left(\tilde{\Omega}_{X}^{p}(\log B)\right)\right) \Rightarrow H^{p+q}\left(\tilde{\Omega}_{X}^{\bullet}(\log B)\right)
$$

degenerates at $E_{1}$, and the cohomology group $H^{n}\left(H_{\mathbf{Q}}\right)$ underlies a mixed Hodge Q-structure for any $n \geq 0$.

Example 3.5. Assume that $X=C_{1} \cup C_{2}, C_{1} \cong C_{2} \cong \mathbf{P}^{1}, C_{1} \cap C_{2}=\left\{P_{1}, P_{2}\right\}$, and $B=0$. Then

$$
\begin{aligned}
& \operatorname{Gr}_{F}^{0}\left(\tilde{\Omega}_{X}^{\bullet}\right)=\tilde{\Omega}_{X}^{0} \cong \mathcal{O}_{C_{1}} \oplus \mathcal{O}_{C_{2}} \\
& \operatorname{Gr}_{F}^{1}\left(\tilde{\Omega}_{X}^{\bullet}\right)=\tilde{\Omega}_{X}^{1}[-1] \\
& =\operatorname{Ker}\left\{\Omega_{C_{1}}^{1}\left(\log \left(P_{1}+P_{2}\right)\right) \oplus \Omega_{C_{2}}^{1}\left(\log \left(P_{1}+P_{2}\right)\right) \rightarrow \mathcal{O}_{P_{1}} \oplus \mathcal{O}_{P_{2}}\right\}[-1] \\
& \cong \omega_{X}[-1]
\end{aligned}
$$

and

$$
\begin{aligned}
& \operatorname{Gr}_{0}^{W}\left(\tilde{\Omega}_{X}^{\bullet}\right)=\left\{\mathcal{O}_{C_{1}} \oplus \mathcal{O}_{C_{2}} \rightarrow \Omega_{C_{1}}^{1} \oplus \Omega_{C_{2}}^{1}\right\} \\
& \operatorname{Gr}_{1}^{W}\left(\tilde{\Omega}_{X}^{\bullet}\right)=\operatorname{Ker}\left\{\left(\mathcal{O}_{P_{1}}^{2} \oplus \mathcal{O}_{P_{2}}^{2}\right)[-1] \rightarrow\left(\mathcal{O}_{P_{1}} \oplus \mathcal{O}_{P_{2}}\right)[-1]\right\} \\
& \cong\left(\mathcal{O}_{P_{1}} \oplus \mathcal{O}_{P_{2}}\right)[-1]
\end{aligned}
$$

Therefore we have the following non-zero cohomology groups:

$$
\operatorname{dim} H^{0}\left(\operatorname{Gr}_{F}^{0}\left(\tilde{\Omega}_{X}^{\bullet}\right)\right)=2, \operatorname{dim} H^{1}\left(\operatorname{Gr}_{F}^{1}\left(\tilde{\Omega}_{X}^{\bullet}\right)\right)=1, \operatorname{dim} H^{2}\left(\operatorname{Gr}_{F}^{1}\left(\tilde{\Omega}_{X}^{\bullet}\right)\right)=1
$$

and

$$
\operatorname{dim} H^{0}\left(\operatorname{Gr}_{0}^{W}\left(\tilde{\Omega}_{X}^{\bullet}\right)\right)=2, \operatorname{dim} H^{1}\left(\operatorname{Gr}_{1}^{W}\left(\tilde{\Omega}_{X}^{\bullet}\right)\right)=2, \operatorname{dim} H^{2}\left(\operatorname{Gr}_{0}^{W}\left(\tilde{\Omega}_{X}^{\bullet}\right)\right)=2 .
$$




\section{Relative case}

We shall extend the construction of the previous section to the relative setting.

We consider the situation of the main theorem. We set $Y^{o}=Y \backslash C, X^{o}=$ $f^{-1}\left(Y^{o}\right), f^{o}=\left.f\right|_{X^{o}}$ and $B^{o}=B \cap X^{o}$. Thus $f^{o}$ is a smooth and projective morphism. We set $X^{o[q]}=X^{[q]} \cap X^{o}$ and $B^{o[q]}=B^{[q]} \cap X^{o}$ with open immersions $i_{q}: X^{o[q]} \backslash B^{o[q]} \rightarrow X^{o[q]}$.

We define the relative De Rham complex

$$
\tilde{\Omega}_{X^{o} / Y^{o}}\left(\log B^{o}\right)
$$

to be the quotient of a differential graded algebra $\tilde{\Omega}_{X^{o}}\left(\log B^{o}\right)$ by an ideal generated by a locally free subsheaf $f^{*} \Omega_{Y^{o}}^{1}$ of $\tilde{\Omega}_{X^{o}}^{1}\left(\log B^{o}\right)$. Then

$$
\tilde{\Omega}_{X^{o} / Y^{o}}^{\operatorname{dim} X-\operatorname{dim} Y}\left(\log B^{o}\right) \cong \omega_{X^{o} / Y^{o}}\left(B^{o}\right)
$$

is an invertible sheaf. The residue exact sequence becomes

$$
\begin{aligned}
0 & \rightarrow \tilde{\Omega}_{X^{o} / Y^{o}}^{\bullet}\left(\log B^{o}\right) \rightarrow \Omega_{X^{o[0]} / Y^{o}}\left(\log B^{o[0]}\right) \rightarrow \Omega_{X^{o[1]} / Y^{o}}^{\bullet}\left(\log B^{o[1]}\right)[-1] \\
& \rightarrow \Omega_{X^{o[2]} / Y^{o}}\left(\log B^{o[2]}\right)[-2] \rightarrow \ldots
\end{aligned}
$$

where the complexes $\Omega_{X^{o[p]} / Y^{o}}^{\bullet}\left(\log B^{o[p]}\right)$ are defined similarly.

The weight filtration on the complex $\tilde{\Omega}_{X^{o} / Y^{o}}\left(\log B^{o}\right)$ is defined by the following exact sequence

$$
\begin{aligned}
& 0 \rightarrow W_{q}\left(\tilde{\Omega}_{X^{o} / Y^{o}}^{\bullet}\left(\log B^{o}\right)\right) \rightarrow W_{q}\left(\Omega_{X^{o[0]} / Y^{o}}^{\bullet}\left(\log B^{o[0]}\right)\right) \\
& \rightarrow W_{q-1}\left(\Omega_{X^{o[1]} / Y^{o}}^{\bullet}\left(\log B^{o[1]}\right)[-1]\right) \rightarrow W_{q-2}\left(\Omega_{X^{o[2]} / Y^{o}}^{\bullet}\left(\log B^{o[2]}\right)[-2]\right) \rightarrow \ldots
\end{aligned}
$$

where the $W$ 's from the second terms denote the filtration with respect to the order of log poles, and the Hodge filtration by

$$
F^{p}\left(\tilde{\Omega}_{X^{o} / Y^{o}}^{\bullet}\left(\log B^{o}\right)\right)=\tilde{\Omega}_{X^{o} / Y^{o}}^{\geq p}\left(\log B^{o}\right) .
$$

Let $i_{q}^{o}: X^{o[q]} \backslash B^{o[q]} \rightarrow X^{o[q]}$ denote an open immersion for any $q$. Then we have

$$
R\left(i_{q}^{o}\right)_{*} \mathbf{Q}_{X^{o[q]} \backslash B^{o[q]}} \otimes\left(f^{o}\right)^{-1} \mathcal{O}_{Y^{o}} \cong \tilde{\Omega}_{X^{o[q]} / Y^{o}}\left(\log B^{o[q]}\right)
$$


We deduce that there exists a convolution $H_{\mathbf{Q}}^{o}$ of the following complex of objects on $X^{o}$ :

$$
\begin{aligned}
& R\left(i_{0}^{o}\right)_{*} \mathbf{Q}_{X^{o[0]} \backslash B^{o[0]}} \rightarrow R\left(i_{1}^{o}\right)_{*} \mathbf{Q}_{X^{o[1]} \backslash B^{o[1]}}[-1] \\
& \rightarrow R\left(i_{2}^{o}\right)_{*} \mathbf{Q}_{X^{o[2]} \backslash B^{o[2]}}[-2] \rightarrow \ldots
\end{aligned}
$$

such that

$$
H_{\mathbf{Q}}^{o} \otimes\left(f^{o}\right)^{-1} \mathcal{O}_{Y^{o}} \cong \tilde{\Omega}_{X^{o} / Y^{o}}\left(\log B^{o}\right)
$$

as in the previous section.

Since our family $f^{o}$ is topologically locally trivial, the higher direct images

$$
R^{p} f_{*}^{o} R\left(i_{q}^{o}\right)_{*} \mathbf{Q}_{X^{o[q]} \backslash B^{o[q]}}
$$

are locally constant sheaves of $\mathbf{Q}$-modules on $Y^{o}$ for all $p$ and $q$, and the homomorphisms between them are flat. It follows that the sheaf $R^{n} f_{*}^{o} H_{\mathbf{Q}}^{o}$ is also locally constant for any $n$.

In a similar way as before, we define a weight filtration $W_{q}\left(H_{\mathbf{Q}}\right)$ on $H_{\mathbf{Q}}$ as convolutions of the following complexes:

$$
\begin{aligned}
& \left.\tau_{\leq q}\left(R\left(i_{0}^{o}\right)_{*} \mathbf{Q}_{X^{o[0]} \backslash B^{o[0]}}\right) \rightarrow \tau_{\leq q}\left(i_{1}^{o}\right)_{*} \mathbf{Q}_{X^{o[1]} \backslash B^{o[1]}}[-1]\right) \\
& \left.\rightarrow \tau_{\leq q}\left(i_{2}^{o}\right)_{*} \mathbf{Q}_{X^{o[2]} \backslash B^{o[2]}}[-2]\right) \rightarrow \ldots
\end{aligned}
$$

such that

$$
W_{q}\left(H_{\mathbf{Q}}^{o}\right) \otimes\left(f^{o}\right)^{-1} \mathcal{O}_{Y^{o}} \cong W_{q}\left(\tilde{\Omega}_{X^{o} / Y^{o}}\left(\log B^{o}\right)\right) .
$$

The restrictions of above constructions to the fibers of $f$ coincide with those in the previous section.

The transversality of the natural connection $\nabla$ on $R^{n} f_{*}^{o}\left(\tilde{\Omega}_{X^{o} / Y^{o}}\left(\log B^{o}\right)\right)$ to the Hodge filtration follows from the topological local triviality of $f^{o}$.

Therefore we obtain the following:

\section{Theorem 4.1.}

$$
\left(\left(R^{n} f_{*}^{o} H_{\mathbf{Q}}^{o}, W\right),\left(R^{n} f_{*}^{o}\left(\tilde{\Omega}_{X^{o} / Y^{o}}\left(\log B^{o}\right)\right), W, F\right)\right)
$$

is a variation of mixed Hodge $\mathbf{Q}$-structures on $Y^{o}$ for any $n$.

Corollary 4.2. The weight spectral sequence

$$
{ }_{W} E_{1}^{p, q}=R^{p+q} f_{*} G r_{-p}^{W}\left(H_{\mathbf{Q}}\right) \Rightarrow R^{p+q} f_{*}\left(H_{\mathbf{Q}}\right)
$$


degenerates at $E_{2}$, and the Hodge spectral sequence

$$
{ }_{F} E_{1}^{p, q}=R^{q} f_{*}\left(\tilde{\Omega}_{X^{o} / Y^{o}}^{p}\left(\log B^{o}\right)\right) \Rightarrow R^{p+q} f_{*} f^{-1} \mathcal{O}_{Y^{o}}
$$

degenerates at $E_{1}$.

\section{Semipositivity theOREM}

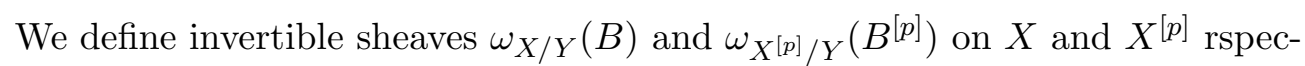
tively by

$$
\omega_{X / Y}(B)=\omega_{X}(B) \otimes f^{*} \omega_{Y}, \quad \omega_{X[p] / Y}\left(B^{[p]}\right)=\omega_{X[p]}\left(B^{[p]}\right) \otimes f^{*} \omega_{Y} .
$$

Then the residue homomorphisms yield an exact sequence of sheaves on $X$ (not only on $X^{o}$ ):

$$
0 \rightarrow \omega_{X / Y}(B) \rightarrow \omega_{X}^{[0] / Y}\left(B^{[0]}\right) \rightarrow \omega_{X}^{[1] / Y}\left(B^{[1]}\right) \rightarrow \omega_{X^{[2]} / Y}\left(B^{[2]}\right) \rightarrow \ldots
$$

The semipositivity theorem (Theorem 1.1) follows from the combination of the following theorem with a result in [9]:

Theorem 5.1. Let $\tilde{\mathcal{H}}^{n}$ be the canonical extension of the variation of mixed Hodge structures $\mathcal{H}^{n}=R^{n} f_{*}^{o}\left(\tilde{\Omega}_{X^{o} / Y^{o}}\left(\log B^{o}\right)\right)$, and let $\tilde{F}$ be the extended Hodge filtration on $\tilde{\mathcal{H}}^{n}$. Then there is an isomorphism

$$
R^{q} f_{*} \omega_{X / Y}(B) \cong F^{d(X / Y)}\left(\tilde{\mathcal{H}}^{q+d(X / Y)}\right)
$$

where $d(X / Y)=\operatorname{dim} X-\operatorname{dim} Y$.

Proof. We put a weight filtration on $\omega_{X / Y}(B)$ by exact sequences

$$
\begin{aligned}
0 & \rightarrow W_{q}\left(\omega_{X / Y}(B)\right) \rightarrow W_{q}\left(\omega_{X}^{[0] / Y}\left(B^{[0]}\right)\right) \\
& \rightarrow W_{q-1}\left(\omega_{X^{[1]} / Y}\left(B^{[1]}\right)\right) \rightarrow W_{q-2}\left(\omega_{X^{[2]} / Y}\left(B^{[2]}\right)\right) \rightarrow \ldots
\end{aligned}
$$

where the $W$ from the second terms are filtrations with respect to the order of $\log$ poles. It is the part of the highest Hodge filtrations of the weight filtration in the previous section if we restrict the sheaves over $X^{o}$ and shift the degree by $-d(X / Y)$ in the sense that

$$
F^{d(X / Y)-p} W_{q-p}\left(\Omega_{X^{o[p]} / Y^{o}}\left(\log B^{o[p]}\right)[-p]\right) \cong W_{q-p}\left(\omega_{X^{o[p]} / Y^{o}}\left(B^{o[p]}\right)\right)[-d(X / Y)] .
$$


Let $X^{[p],[q]}$ be the disjoint union of all the closed strata of the pair $(X, B)$ which are contained in $X^{[p]}$ and has codimension $q$ in $X^{[p]}$. Then we have

$$
\begin{aligned}
& \operatorname{Gr}_{q}^{W}\left(\omega_{X[p] / Y}\left(B^{[p]}\right)\right) \cong \omega_{X[p],[q] / Y} \\
& \operatorname{Gr}_{q}^{W}\left(\omega_{X / Y}(B)\right) \cong \bigoplus_{\operatorname{dim} X-\operatorname{dim} Z=q} \omega_{Z / Y}
\end{aligned}
$$

as before.

By Corollary 4.2 and Lemma 3.2, we have a weight spectral sequence

$$
{ }_{W} E_{1}^{p, q}=\bigoplus_{\operatorname{dim} X-\operatorname{dim} Z=-p} R^{2 p+q} f_{*}^{o} \Omega_{Z^{o} / Y^{o}} \Rightarrow R^{p+q} f_{*}^{o}\left(\tilde{\Omega}_{X^{o} / Y^{o}}^{\bullet}\left(\log B^{o}\right)\right)
$$

which degenerates at $E_{2}$, where we put $Z^{o}=Z \cap X^{o}$. The boundary homomorphism $d_{1}^{p, q}$ of the spectral sequence (5.1) at the $E_{1}$-level is given by the sum of the connecting homomorphisms

$$
R^{2 p+q} f_{*}^{o} \Omega_{Z^{o} / Y^{o}}^{\bullet} \rightarrow R^{2 p+q+2} f_{*}^{o} \Omega_{\left(Z^{\prime}\right)^{o} / Y^{o}}^{\bullet}
$$

of the exact sequences

$$
0 \rightarrow \Omega_{\left(Z^{\prime}\right)^{\circ} / Y^{o}}^{\bullet} \rightarrow \Omega_{\left(Z^{\prime}\right)^{o} / Y^{o}}\left(\log Z^{o}\right) \rightarrow \Omega_{Z^{\circ} / Y^{o}}^{\bullet}[-1] \rightarrow 0
$$

for the inclusions $Z \subset Z^{\prime}$ of the closed strata of the pair $(X, B)$ such that $\operatorname{dim} Z=$ $\operatorname{dim} X+p$ and $\operatorname{dim} Z^{\prime}=\operatorname{dim} X+p+1$, where we put $\left(Z^{\prime}\right)^{o}=Z^{\prime} \cap X^{o}$.

Let $\tilde{\mathcal{H}}_{Z}^{2 p+q}$ be the canonical extensions of the variations of Hodge structures $\mathcal{H}_{Z}^{2 p+q}=R^{2 p+q} f_{*}^{o} \Omega_{Z^{\circ} / Y^{o}}$ for the closed strata $Z$ of codimension $-p$. Since the formation of canonical extensions is functorial, (5.1) extends to another spectral sequence

$$
{ }_{W} \tilde{E}_{1}^{p, q}=\bigoplus_{\operatorname{dim} X-\operatorname{dim} Z=-p} \tilde{\mathcal{H}}_{Z}^{2 p+q} \Rightarrow \tilde{\mathcal{H}}^{p+q}
$$

It is known by [12] and [13] (and [9] if $q=0$ ) that the highest Hodge parts of the canonical extensions are isomorphic to the higher direct images of dualizing sheaves:

$$
F^{p+d(X / Y)}\left(\tilde{\mathcal{H}}_{Z}^{2 p+q}\right) \cong R^{p+q-d(X / Y)} f_{*} \omega_{Z / Y} .
$$

In particular, the right hand side are locally free sheaves on $Y$. 
On the other hand, we have another spectral sequence with respect to the weight filtration

$$
{ }_{W} \bar{E}_{1}^{p, q}=\bigoplus_{\operatorname{dim} X-\operatorname{dim} Z=-p} R^{p+q} f_{*} \omega_{Z / Y} \Rightarrow R^{p+q} f_{*}\left(\omega_{X / Y}(B)\right)
$$

so that

$$
{ }_{W} \bar{E}_{1}^{p, q-d(X / Y)} \cong F^{p+d(X / Y)}\left({ }_{W} \tilde{E}_{1}^{p, q}\right) .
$$

The boundary homomorphism $\bar{d}_{1}^{p, q}$ of (5.4) is given by the sum of the connecting homomorphisms

$$
R^{p+q} f_{*} \omega_{Z / Y} \rightarrow R^{p+q+1} f_{*} \omega_{Z^{\prime} / Y}
$$

of the adjunction sequences

$$
0 \rightarrow \omega_{Z^{\prime} / Y} \rightarrow \omega_{Z^{\prime} / Y}(Z) \rightarrow \omega_{Z / Y} \rightarrow 0
$$

The homomorphism (5.2) is a morphism of variations of Hodge structures of bidegree $(1,1)$. If we take the parts of the highest Hodge fitrations in $(5.2)$, then we obtain a homomorphism

$$
R^{p+q-d(X / Y)} f_{*}^{o} \omega_{Z^{o} / Y^{o}} \rightarrow R^{p+q+1-d(X / Y)} f_{*}^{o} \omega_{\left(Z^{\prime}\right)^{o} / Y^{o}}
$$

which coincides with the restriction of the homomorphism (5.5) to $Y^{o}$ if we shift the degree by $d(X / Y)$. Therefore the $E_{2}$ terms of the spectral sequence (5.1) are also variations of Hodge structures, and we have

$$
\left.F^{p+d(X / Y)}\left({ }_{W} E_{2}^{p, q}\right) \cong{ }_{W} \bar{E}_{2}^{p, q-d(X / Y)}\right|_{Y^{o}} .
$$

It follows also that the canonical extensions of the ${ }_{W} E_{2}^{p, q}$ coincide with the ${ }_{W} \tilde{E}_{2}^{p, q}$, and

$$
F^{p+d(X / Y)}\left({ }_{W} \tilde{E}_{2}^{p, q}\right) \cong{ }_{W} \bar{E}_{2}^{p, q-d(X / Y)} .
$$

We already know that the boundary homomorphisms $d_{m}^{p, q}$ of the spectral sequence (5.1) vanish for $m \geq 2$ and all $p, q$. Since the sheaves ${ }_{W} \tilde{E}_{2}^{p, q}$ and ${ }_{W} \bar{E}_{2}^{p, q}$ are locally free, it follows that $\tilde{d}_{2}^{p, q}=\bar{d}_{2}^{p, q}=0$ for the corresponding boundary homomorphisms of the spectral sequences (5.3) and (5.4), respectively, hence $\tilde{d}_{m}^{p, q}=\bar{d}_{m}^{p, q}=0$ for $m \geq 2$ and all $p, q$, and we obtain the theorem.

Let $L$ be the polarization of the total space $X$ given by the cohomology class of an ample line bundle. We define the primitive parts of the cohomology groups thoughout the argument as the kernels with respect to the operation taking the cup product with $L$. Since this operation is compatible with all other operations, 
our variations of mixed $\mathbf{Q}$-Hodge structures become graded polarizable. We note that the highest Hodge parts are not affected by this operation because they always vanish when multiplied by $L$. By the semipositivity theorem of [9], the sheaves $F^{p+d(X / Y)}\left({ }_{W} \tilde{E}_{2}^{p, q}\right)$ are numerically semipositive. Since $R^{q} f_{*} \omega_{X / Y}(B)$ is an extension of these sheaves, it is also numerically semipositive. This is the conclusion of the proof of Theorem 1.1.

\section{REFERENCES}

[1] Abramovich, D.; Karu, K. Weak semistable reduction in characteristic 0. Invent. Math. 139 (2000), no. 2, 241-273.

[2] Berndtsson, Bo; Paun, Mihai. Bergman kernels and the pseudoeffectivity of relative canonical bundles. Duke Math. J. 145 (2008), no. 2, 341-378.

[3] Deligne, Pierre. Théorie de Hodge III. Inst. Hautes Etudes Sci. Publ. Math. No. 44 (1974), $5-77$.

[4] Du Bois, Philippe. Complexe de de Rham filtre d'une variete singuliere. Bull. Soc. Math. France 109 (1981), no. 1, 41-81.

[5] Fujino, Osamu. Higher direct images of log canonical divisors. J. Differential Geom. 66 (2004), no. 3, 453-479.

[6] Fujita, Takao. On Kähler fiber spaces over curves. J. Math. Soc. Japan 30 (1978), no. 4, 779-794.

[7] Gelfand, Sergei I.; Manin, Yuri I. Methods of Homological Algebra. Springer Verlag, 1997.

[8] Griffiths, Phillip A. Periods of integrals on algebraic manifolds. III. Some global differentialgeometric properties of the period mapping. Inst. Hautes Etudes Sci. Publ. Math. No. 381970 $125-180$.

[9] Kawamata, Yujiro. Characterization of abelian varieties. Compositio Math. 43 (1981), 253276.

[10] Kawamata, Yujiro. Subadjunction of log canonical divisors. II. alg-geom/9712014. Amer. J. Math. 120 (1998), no. 5, 893-899.

[11] Kawamata, Yujiro. On algebraic fiber spaces. math.AG/0107160. in Contemporary Trends in Algebraic Geometry and Algebraic Topology, World Scientific, 2002, 135-154.

[12] Kollár, János. Higher direct images of dualizing sheaves. II. Ann. of Math. (2) 124 (1986), no. 1, 171-202.

[13] Nakayama, Noboru. Hodge filtrations and the higher direct images of canonical sheaves. Invent. Math. 85 (1986), no. 1, 217-221.

[14] Schmid, Wilfried. Variation of Hodge structure: the singularities of the period mapping. Invent. Math. 22 (1973), 211-319.

[15] Steenbrink, Joseph; Zucker, Steven. Variation of mixed Hodge structure. I. Invent. Math. 80 (1985), no. 3, 489-542. 
Yujiro Kawamata

Department of Mathematical Sciences, University of Tokyo,

Komaba, Meguro, Tokyo, 153-8914, Japan

E-mail: kawamata@ms.u-tokyo.ac.jp 